\title{
Assessment of the Accuracy of a Bayesian Estimation Algorithm for Perfusion CT by Using a Digital Phantom
}

Makoto Sasaki, MD, $\mathrm{PhD}^{1}$; Kohsuke Kudo, MD, $\mathrm{PhD}^{1}$; Timothé Boutelier, $\mathrm{PhD}^{2}$; Fabrice Pautot, $\mathrm{PhD}^{2}$; Soren Christensen, $\mathrm{PhD}^{3}$, Ikuko Uwano, $\mathrm{PhD}^{1}$; Jonathan Goodwin, $\mathrm{PhD}^{1}$; Satomi Higuchi, $\mathrm{PhD}^{1}$; Kenji Ito, $\mathrm{PhD}^{1}$; Fumio Yamashita, $\mathrm{PhD}^{1}$

${ }^{1}$ Division of Ultrahigh Field MRI, Institute for Biomedical Sciences, Iwate Medical University, Yahaba, Japan

${ }^{2}$ Department of Research \& Innovation, Olea Medical, La Ciotat, France

${ }^{3}$ Department of Neurology and Radiology, University of Melbourne, Royal Melbourne Hospital, Victoria, Australia

\author{
Corresponding Author \\ Makoto Sasaki, MD, PhD \\ Division of Ultrahigh Field MRI \\ Institute for Biomedical Sciences \\ Iwate Medical University \\ 2-1-1 Nishitokuta \\ Yahaba 028-3694
}


Japan

Tel: +81-19-651-5111

Fax: +81-19-908-8021

e-mail: masasaki@iwate-med.ac.jp 


\section{Abstract}

Introduction: A new deconvolution algorithm, the Bayesian estimation algorithm, was reported to improve the precision of parametric maps created using perfusion computed tomography. However, it remains unclear whether quantitative values generated by this method are more accurate than those generated using optimized deconvolution algorithms of other software packages. Hence, we compared the accuracy of the Bayesian and deconvolution algorithms by using a digital phantom.

Methods: The digital phantom data, in which concentration-time curves reflecting various known values for cerebral blood flow (CBF), cerebral blood volume (CBV), mean transit time (MTT), and tracer delays were embedded, were analyzed using the Bayesian estimation algorithm as well as delay-insensitive singular value decomposition (SVD) algorithms of 2 software packages that were the best benchmarks in a previous cross-validation study. Correlation and agreement of quantitative values of these algorithms with true values were examined.

Results: CBF, CBV, and MTT values estimated by all the algorithms showed strong correlations with the true values $(r=0.91-0.92,0.97-0.99$, and 0.91-0.96, respectively). In addition, the values generated by the Bayesian estimation algorithm for all of these parameters showed good agreement with the true values (intraclass correlation coefficient $[\mathrm{ICC}]=0.90,0.99$, and 0.96 , respectively), while MTT values from the SVD algorithms were suboptimal $(\mathrm{ICC}=0.81-0.82)$. 
Conclusions: Quantitative analysis using a digital phantom revealed that the Bayesian estimation algorithm yielded CBF, CBV, and MTT maps strongly correlated with the true values and MTT maps with better agreement than those produced by delay-insensitive SVD algorithms.

\section{Keywords:}

perfusion computed tomography, digital phantom, Bayesian estimation algorithm, mean transit time, cerebral blood flow 


\section{Introduction}

Perfusion computed tomography (PCT) is widely used to estimate the extent of the hypoperfused area with ischemic penumbra in patients with acute stroke [1]. However, software packages and algorithms have been reported to yield substantially different PCT maps and quantitative values for acute stroke patients; such differences should be minimized [2-5]. To resolve this issue, the Stroke Imaging Repository (STIR) Consortium examined the accuracy and reliability of various post-processing programs by using a sophisticated digital phantom [6]. The study revealed strong correlations between values generated from some delay-insensitive singular value decomposition (SVD) deconvolution algorithms implemented in some software packages and true values embedded in the phantom with respect to cerebral blood flow (CBF), cerebral blood volume (CBV), and mean transit time (MTT) maps. Even with these algorithms, however, agreement between true values and CBF and/or MTT values was imperfect. Recently, another new algorithm, based on commonly-used Bayesian probabilistic approach to parameter estimation, has been introduced as more accurate than deconvolution methods for estimating CBF and MTT maps [7,8]. This method can directly estimate the residue function of brain tissues, $R(t)$, by applying Bayesian probability theory on the intravascular tracer model and can calculate other perfusion metrics by reconvoluting $R(t)$. Hence, this technique is inherently delay-insensitive and can robustly estimate hemodynamic parameters so that it potentially overcome known limitations of the SVD algorithm family, such as non-physiological oscillation of estimated $R(t)$ and vulnerability to the image noise that is 
characteristic of PCT [7-10]. However, it remains unclear whether this algorithm can improve the precision of quantitative values compared with other optimized algorithms of software packages that have been identified as the best benchmarks.

\section{Aims}

We compared the accuracy of the Bayesian estimation algorithm with that of other optimized deconvolution programs by using the digital phantom to determine advantages of the Bayesian estimation algorithm in terms of correlations and agreements with ground truth values.

\section{Materials and Methods}

\section{Digital Phantom}

We used the digital phantom that was introduced in a previous study [6]. Briefly, concentration-time curves for the artery (arterial input function, AIF), vein (venous output function, VOF), and brain tissue were created using a round robin of variable parameters, as follows: 3 kinds of $R(t)$ (exponential, linear, and box shaped), 7 MTT values (3.4, 4.0, 4.8, 6.0, 8.0, 12.0, and 24.0 s), 5 CBV values (1.0, 2.0, 3.0, 4.0, and 5.0 mL/100 g), and 7 values of delay in tracer arrival $(0.0,0.5,1.0,1.5,2.0,2.5$, and $3.0 \mathrm{~s})$. All curves were then sampled with a time interval of $2 \mathrm{~s}$ and a duration of 60 s (30 phases) after converting to Hounsfield units to mimic PCT source data. These values were embedded in the digital phantom data set that 
contained 16 slice locations; slice 1 contained the AIF and VOF and slices $2-16$ contained tissue curves within $7 \times 7$ quadratic tiles of $32 \times 32$ pixels that were located in a real brain CT image (Figure 1). These data were exported to Digital Imaging and Communication in Medicine (DICOM) format after Gaussian noise was added for a signal-to-noise ratio (SNR) of 5.0, which is roughly equivalent to the SNR in clinical PCT data scanned with $80 \mathrm{kVp}$ and $200 \mathrm{mAs}$.

\section{Data Analysis}

These phantom data were post-processed using a development version of Olea Sphere (Olea Medical; La Ciotat, France) with the Bayesian estimation algorithm [7] as well as with a commercially available software package (CBP Study Ph8; Toshiba Medical Systems; Tokyo, Japan) and a program developed by an academic institution (Perfusion Mismatch Analyzer, PMA; Acute Stroke Imaging Standardization Group Japan [ASIST-Japan]; http://asist.umin.jp/index-e.htm). We selected CBP Study Ph8 and PMA among various commercial and academic software packages because these programs formed the best benchmark in a previous digital phantom validation study [6]. The former implemented a reformulated SVD (rSVD) algorithm, called as SVD+, that can allow the perfusion metrics to be calculated with insensitivity to tracer delay by shifting AIF [11], while the latter implemented a block-circulant SVD (bSVD) algorithm that can minimize the sensitivity of tracer arrival difference between AIF and brain tissue by using a block circulant matrix [9]. CBF, CBV, and MTT maps generated by each software program were exported 
to DICOM format and were then loaded and displayed in the PMA software for further analyses because PMA enabled to perform automated multiple ROI measurements. The average pixel value for each tile within the maps was automatically measured with the PMA software and a square region of interest $(\mathrm{ROI})$ of $28 \times 28$ pixels.

Pearson's correlation coefficient $(r)$ was calculated for the correlation between values obtained with each program and true values (CBF, CBV, and MTT). In addition, intraclass correlation coefficient (ICC) was used to evaluate agreement with true values. Slope (a) and intercept (b) of linear regression $(y=a x+b)$ were also calculated to evaluate agreement of the measured values $(x)$ with the true values $(y)$. A repeated-measures analysis of variance (ANOVA) was used to examine dependencies of CBF, CBV, and MTT values in each program on tracer delay values.

\section{Results}

CBF, CBV, and MTT maps were successfully generated using all of the programs (Figure 2). The color maps of CBF and MTT showed distinct gradients, and color maps of CBV were nearly consistent in the horizontal direction, which is generally comparable to the true values. In addition, all the maps showed no gradient in the vertical direction, indicating that all the algorithms are independent of the tracer delay.

Bayesian, rSVD, and bSVD algorithms resulted in strong correlation with the true values of CBF ( $r=0.91,0.92$, and 0.92 , respectively), CBV ( $r$ 
$=0.99,0.97$, and 0.98, respectively), and MTT ( $r=0.96,0.95$, and 0.91, respectively) (Table 1, Figure 3). In addition, the ICCs of CBV values were 0.99, 0.97, and 0.98 for the Bayesian, rSVD, and bSVD algorithms, respectively, indicating good agreement with true values. CBF and MTT values generated with the Bayesian algorithm also showed good agreement (ICC $=0.90$ and 0.96, respectively). However, agreement of CBF and MTT values generated with $\mathrm{rSVD}$ (ICC $=0.87$ and 0.81 , respectively) and agreement of MTT values generated with $\mathrm{bSVD}(\mathrm{ICC}=0.82)$ were insufficient (Table 1, Figure 3).

Regarding the regression lines, the slopes (a) and intercepts (b) of CBV were nearly ideal $(y=x)$ with Bayesian, rSVD, and bSVD algorithms (a $=1.02,1.04$, and $0.92 ; \mathrm{b}=-0.04,-0.31$, and -0.23 ; respectively). However, the slopes and intercepts of CBF and MTT in rSVD $(\mathrm{a}=0.64$ and 0.54 , $\mathrm{b}=3.44$ and 4.09 , respectively) and $\mathrm{bSVD}(\mathrm{a}=1.07$ and $0.58, \mathrm{~b}=7.37$ and 4.74 , respectively) algorithms were suboptimal, and the values were overestimated in the lower ranges and/or underestimated in the higher ranges. In contrast, the slopes and intercepts of CBF and MTT in the Bayesian algorithm were close to 1 and 0 , respectively $(a=1.12$ and $0.86, b=-1.00$ and 1.26 , respectively) (Table 1, Figure 3). Bayesian and bSVD algorithms showed no significant differences in CBF, CBV, and MTT values among the different delay values $(p=0.38-054$ and $0.10-0.70$, respectively; repeated-measures ANOVA) indicating being insensitive to the tracer delay, while rSVD algorithm showed significant differences in CBF and MTT values ( $<$ 0.001) (Table 1). 


\section{Discussion}

Bolus-tracking cerebral perfusion imaging such as PCT and perfusion-weighted magnetic resonance imaging (PWI) is widely utilized to evaluate the extent of ischemic penumbral areas in acute stroke patients who are candidates for recanalization therapies, particularly beyond $4.5 \mathrm{~h}$ after onset. However, a recent trial using PCT or PWI as one of the entry criteria could not determine the efficacy of a thrombolytic agent 3-9 h after stroke onset [12]. This failure can in part be attributed to unexpectedly large differences in the perfusion metrics and their quantitative values between the PCT/PWI analysis programs, due to errors mainly caused by delay in tracer arrival [2-5]. To minimize this grave issue, STIR Consortium developed a sophisticated digital phantom and cross-validated various algorithms of all present software packages with the phantom [6]. The study found that with the majority of PCT and PWI software, CBF and MTT values showed substantial delay-induced errors and/or insufficient correlation with true values, although $\mathrm{CBV}$ was generally accurate. In addition, the correlation between PCT and true values tended to be inferior to the correlation between PWI and true values, presumably because of an inherent disadvantage of PCT, such as a low SNR due to the need to reduce radiation exposure. Nevertheless, 2 programs with delay-insensitive SVD algorithms had good results of $r>0.9$ for CBF, CBV, and MTT values. Even with these programs, however, agreement of CBF and MTT with true values was less accurate than agreement of CBV and true values. 
The accuracy of CBF and MTT calculations is affected by many factors related to data acquisition and processing. Injection rate and amount, concentration of contrast agents, cardiac output of patients, scanning parameters, temporal resolution, and scan duration should be considered in analyses of patient data but can be optimized in phantom analyses. Delay in tracer arrival can lead to underestimation of CBF and overestimation of MTT in delay-sensitive algorithms; delay-insensitive algorithms yielded better results in previous studies [2-10]. Delay-insensitive SVD algorithms, which are now commonly used as the most reliable methods, still yield inherent errors with regard to estimating $R(t)$ of hypoperfused tissue due to oscillation, resulting in inaccuracies in CBF and/or MTT calculations $[9,10]$. In addition, an unavoidably low SNR in PCT source images can cause further deterioration in the precision of CBF and MTT estimates. In PCT, CBF and/or MTT, but not CBV, is frequently used to estimate the extent of the penumbral area; hence, it is crucial for this purpose to improve the accuracy of CBF and MTT calculation algorithms.

Recently, the Bayesian estimation algorithm was introduced as an accurate method for estimating $R(t)$ and various perfusion metrics [7,8]. In this algorithm, by applying commonly-used Bayesian probabilistic approach to estimate $R(t)$ of the intravascular tracer model, we can minimize effects of oscillation, tracer delay, and low SNR during estimating $R(t)$ when compared with SVD methods. In addition, quantification of various perfusion metrics such as CBF and MTT becomes more accurate because these parameters are calculated after reconvolution of estimated $R(t)$ with AIF. Although results of simulations suggest potential advantages of Bayesian methods of perfusion imaging analysis, direct comparisons with 
other algorithms under various perfusion conditions have not been achieved. Thus, in this study, the sophisticated digital phantom that was introduced for benchmark study of various analysis programs [6] was applied to further validate the algorithm. Using the phantom, we have objectively shown the accuracy of the Bayesian method for estimating CBF and MTT, in contrast with optimized delay-insensitive SVD programs. In particular, the Bayesian method resulted in an independency on the delay in tracer arrival and a remarkable improvement in the agreement of absolute MTT values with true values. MTT values estimated by the SVD algorithms had suboptimal ICCs and regression lines far different from the ideal lines, i.e., gentle slopes and positive intercepts, which indicates overestimation in lower ranges and underestimation in higher ranges. In contrast, the ICC for MTT values estimated by the Bayesian method was more than 0.95 , and the regression line was almost optimal. These results indicate that the Bayesian algorithm can generate accurate absolute MTT values in wide ranges despite a low SNR, overcoming the inaccuracies in MTT estimation that are inevitable with SVD algorithms. At present, however, feasibility of the Bayesian algorithm remains insufficient because only a few software programs have implemented this algorithm and the postprocessing requires relatively long time when compared with SVD algorithms.

There are several limitations of this study. First, the phantom we used included 3 kinds of $R(t)$, i.e., exponential, linear, and box shaped, because true shapes of $R(t)$ in the healthy and hypoperfused human brain are unknown. However, these shapes are all non-realistic and can induce 
substantial errors or biases in estimation of CBF and MTT with Bayesian and deconvolution methods. Splitting tendencies into 3 ways of Bayesian CBF values which are shown in Figure 3 can be mainly attributed to this issue. Second, this study did not consider errors induced by AIF and dispersion effect, which are known to affect the accuracy of CBF/MTT estimation [13,14]. The phantom used in this study provides only global AIF with the size and shape being suitable for automatic detection. Hence, the difference between algorithms in performance of AIF detection and in errors induced by dispersion effects remains unknown. Third, we did not investigate the dependence of the algorithms on SNR, which is a major constraint on optimization of PCT analysis. Although the phantom included substantial noise equivalent to that of a standard low-dose scan, SNR varies among scanners and scanning parameters. In addition, susceptibility of the algorithms to the noise depends on denoising methods implemented in the programs. Further studies are needed to elucidate advantages of the Bayesian method for low-dose PCT over SVD methods. Another limitation is that this study did not include a comparison of the time-of-maximum of $R(t)$ (Tmax). In PWI, Tmax is considered one of the most reliable of various perfusion parameters [15] and has been used in recent major clinical trials [16-18]. However, only a few PCT analysis programs can calculate Tmax. In addition, the Bayesian method cannot generate Tmax directly, because Tmax is a non-realistic perfusion parameter. Tmax generated by SVD algorithms is considered to include several parameters such as tracer delay, dispersion, and MTT [19]. For direct comparison of Tmax among algorithms, it is necessary to establish methods for calculating Tmax from other perfusion parameters that can be 
obtained using the Bayesian algorithm. Finally, we did not compare PCT data of acute ischemic stroke and other neurological disorders such as brain tumors, because that is beyond the scope of this study. Our forthcoming investigations using data from animal models, patients with acute stroke, and those with brain tumors are necessary to establish the clinical significance of the Bayesian estimation method.

In conclusions, quantitative analyses using a digital phantom revealed that only the Bayesian estimation algorithm generated CBF and MTT values that agreed well with the true values, whereas those generated by the delay-insensitive SVD algorithms were substantially different from the true values. The Bayesian estimation algorithm can generate more accurate PCT maps than SVD algorithms and is considered promising in quantitative analyses of ischemic penumbra in acute stroke patients. 


\section{Acknowledgments}

This work was supported in part by a Grant-in-Aid for Strategic Medical Science Research from the Ministry of Education, Culture, Sports, Science and Technology of Japan.

\section{Conflict of interest}

MS has served on the advisory board for Olea Medical. TB and FP are employees of Olea Medical. 


\section{References}

1. Lev MH, Segal AZ, Farkas J, Hossain ST, Putman C, Hunter GJ, Budzik R, Harris GJ, Buonanno FS, Ezzeddine MA, Chang Y, Koroshetz WJ, Gonzalez RG, Schwamm LH (2001) Utility of perfusion-weighted CT imaging in acute middle cerebral artery stroke treated with intra-arterial thrombolysis: Prediction of final infarct volume and clinical outcome. Stroke 32: 2021-2028

2. Kudo K, Sasaki M, Ogasawara K, Terae S, Ehara S, Shirato H (2009) Difference in tracer delay-induced effect among deconvolution algorithms in CT perfusion analysis: Quantitative evaluation with digital phantoms. Radiology 251: 241-249

3. Kudo K, Sasaki M, Yamada K, Momoshima S, Utsunomiya H, Shirato H, Ogasawara K (2010) Differences in CT perfusion maps generated by different commercial software: Quantitative analysis by using identical source data of acute stroke patients. Radiology 254: 200-209

4. Sasaki M, Kudo K, Ogasawara K, Fujiwara S (2009) Tracer delay-insensitive algorithm can improve reliability of CT perfusion imaging for cerebrovascular steno-occlusive disease: Comparison with quantitative single-photon emission CT. Am J Neuroradiol 30: 188-193

5. Fahmi F, Marquering HA, Streekstra GJ, Beenen LF, Velthuis BK, Vanbavel E, Majoie CB (2012) Differences in CT perfusion summary maps for patients with acute ischemic stroke generated by 2 software packages. Am J Neuroradiol 33: 2074-2080

6. Kudo K, Christensen S, Sasaki M, Ostergaard L, Shirato H, Ogasawara K, Wintermark M, Warach S, Warach (2013) Accuracy and reliability 
assessment of CT and MR perfusion analysis software using a digital phantom. Radiology 267: 201-211

7. Mouridsen K, Friston K, Hjort N, Gyldensted L, Ostergaard L, Kiebel S (2006) Bayesian estimation of cerebral perfusion using a physiological model of microvasculature. Neuroimage 33: 570-579

8. Boutelier T, Kudo K, Pautot F, Sasaki M (2012) Bayesian hemodynamic parameter estimation by bolus tracking perfusion weighted imaging. IEEE Trans Med Imaging 31: 1381-1395

9. Wu O, Ostergaard L, Weisskoff RM, Benner T, Rosen BR, Sorensen AG (2003) Tracer arrival timing-insensitive technique for estimating flow in MR perfusion-weighted imaging using singular value decomposition with a block-circulant deconvolution matrix. Magn Reson Med 50: $164-174$

10. Ostergaard L (2005) Principles of cerebral perfusion imaging by bolus tracking. J Magn Reson Imaging 22: 710-717

11. Hanson EH, Roach CJ, Day KJ, Peters KR, Bradley WG, Jr., Ghosh K, Patton PW, McMurray RC, Orrison WW, Jr (2013) Assessment of the tracer delay effect in whole-brain computed tomography perfusion: Results in patients without known neuroanatomic abnormalities. J Comput Assist Tomogr 37: 212-221

12. Hacke W, Furlan AJ, Al-Rawi Y, Davalos A, Fiebach JB, Gruber F, Kaste M, Lipka LJ, Pedraza S, Ringleb PA, Rowley HA, Schneider D, 
Schwamm LH, Leal JS, Sohngen M, Teal PA, Wilhelm-Ogunbiyi K, Wintermark M, Warach S (2009) Intravenous desmoteplase in patients with acute ischaemic stroke selected by MRI perfusion-diffusion weighted imaging or perfusion CT (DIAS-2): A prospective, randomised, double-blind, placebo-controlled study. Lancet Neurol 8: 141-150

13. van Osch MJ, Vonken EJ, Bakker CJ, Viergever MA (2001) Correcting partial volume artifacts of the arterial input function in quantitative cerebral perfusion MRI. Magn Reson Med 45: 477-485

14. Calamante F, Willats L, Gadian DG, Connelly A (2006) Bolus delay and dispersion in perfusion MRI: implications for tissue predictor models in stroke. Magn Reson Med 55: 1180-1185

15. Christensen S, Mouridsen K, Wu O, Hjort N, Karstoft H, Thomalla G, Rother J, Fiehler J, Kucinski T, Ostergaard L (2009) Comparison of 10 perfusion MRI parameters in 97 sub-6-hour stroke patients using voxel-based receiver operating characteristics analysis. Stroke 40: 2055-2061

16. Albers GW, Thijs VN, Wechsler L, Kemp S, Schlaug G, Skalabrin E, Bammer R, Kakuda W, Lansberg MG, Shuaib A, Coplin W, Hamilton S, Moseley M, Marks MP (2006) Magnetic resonance imaging profiles predict clinical response to early reperfusion: the diffusion and perfusion imaging evaluation for understanding stroke evolution (DEFUSE) study. Ann Neurol 60: 508-517

17. Davis SM, Donnan GA, Parsons MW, Levi C, Butcher KS, Peeters A, Barber PA, Bladin C, De Silva DA, Byrnes G, Chalk JB, Fink JN, Kimber 
TE, Schultz D, Hand PJ, Frayne J, Hankey G, Muir K, Gerraty R, Tress BM, Desmond PM (2008) Effects of alteplase beyond 3 h after stroke in the Echoplanar Imaging Thrombolytic Evaluation Trial (EPITHET): a placebo-controlled randomised trial. Lancet Neurology 7: 299-309

18. Ma H, Parsons MW, Christensen S, Campbell BC, Churilov L, Connelly A, Yan B, Bladin C, Phan T, Barber AP, Read S, Hankey GJ, Markus R, Wijeratne T, Grimley R, Mahant N, Kleinig T, Sturm J, Lee A, Blacker D, Gerraty R, Krause M, Desmond PM, McBride SJ, Carey L, Howells DW, Hsu CY, Davis SM, Donnan GA (2012) A multicentre, randomized, double-blinded, placebo-controlled Phase III study to investigate EXtending the time for Thrombolysis in Emergency Neurological Deficits (EXTEND). Int J Stroke 7: 74-80

19. Calamante F, Christensen S, Desmond PM, Ostergaard L, Davis SM, Connelly A (2010) The physiological significance of the time-to-maximum (Tmax) parameter in perfusion MRI. Stroke 41: 1169-1174 


\section{Figure Legends}

Figure 1. Data structure of the digital phantom simulating various tissue curves

The digital phantom consists of slices in which $7 \times 7$ quadratic tiles of different delay times and mean transit times (MTT) are embedded. CBV (cerebral blood volume) and CBF (cerebral blood flow) values as well as residue function $R(t)$ shapes are different among slices. The phantom also includes a slice that contains the arterial input function and the venous output function (not shown).

Figure 2. Perfusion maps of the digital phantom generated by different algorithms

Slices with cerebral blood volume (CBV) of $5.0 \mathrm{~mL} / 100 \mathrm{~g}$ and exponential $R(t)$. Color maps of cerebral blood flow (CBF), CBV, and mean transit time (MTT) generated by the Bayesian algorithm, reformulated singular value decomposition (rSVD) algorithm, and block-circulant singular value decomposition (bSVD) algorithm appear to be roughly comparable to the true values, although subtle differences between the maps and true values can be seen. No distinct gradation in the vertical direction is found in any of the algorithms, indicating insensitivity to the tracer delay. 
Figure 3. Linear regression analyses of cerebral blood flow (CBF), cerebral blood volume (CBV), and mean transit time (MTT) estimated by Bayesian and SVD algorithms

The Bayesian algorithm showed good correlation and agreement between true values and CBF, CBV, and MTT, while CBF and MTT estimated by reformulated singular value decomposition (rSVD) and MTT estimated by block-circulant singular value decomposition (bSVD) showed insufficient agreement with the true values.

ICC, intraclass correlation coefficient; $r$, correlation coefficient; red broken lines, $y=x$. 


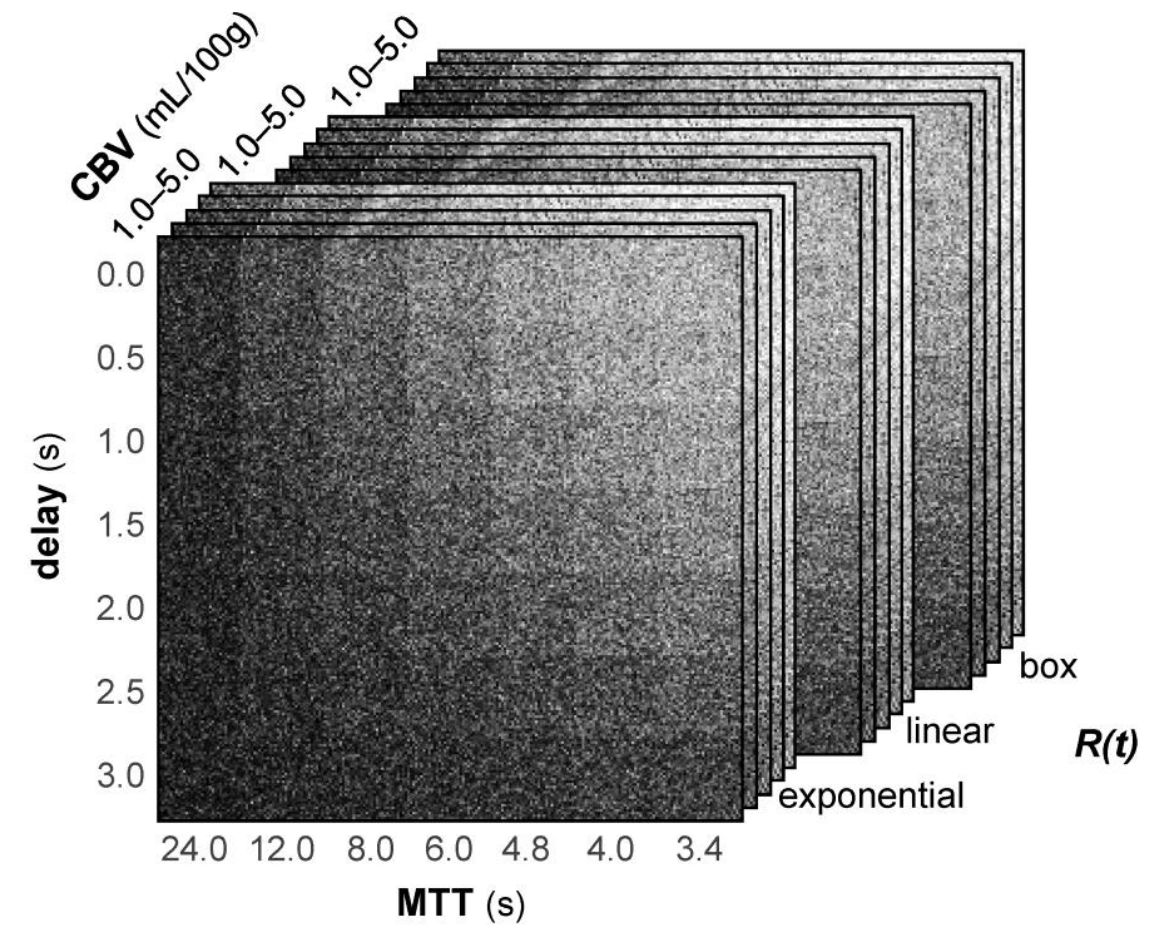




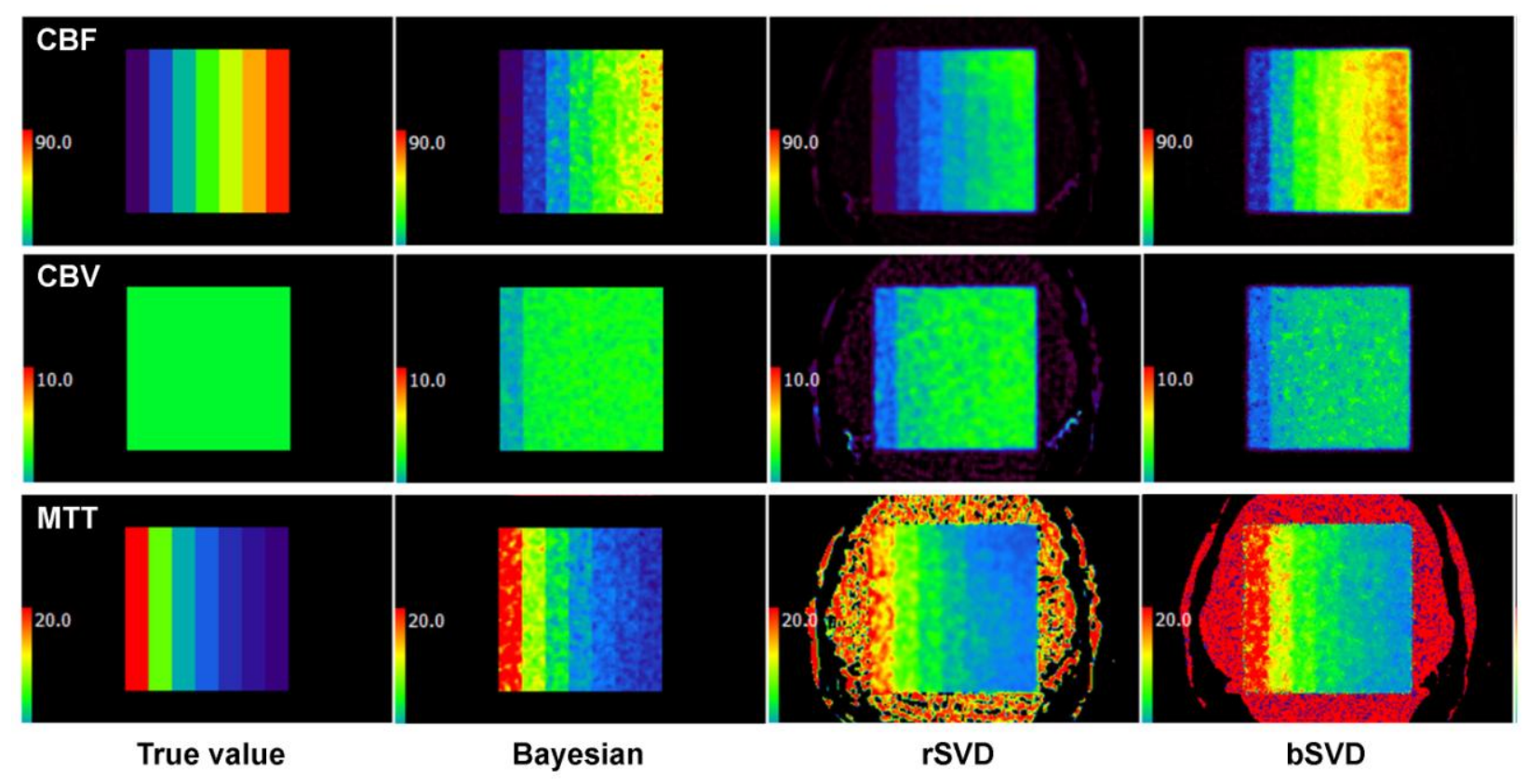


CBF

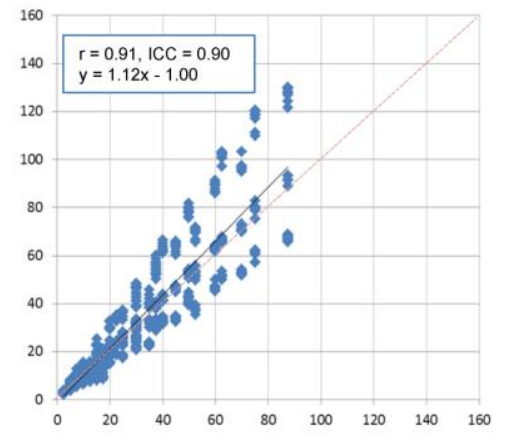

True CBF (mL/100g/min)

\section{CBV}

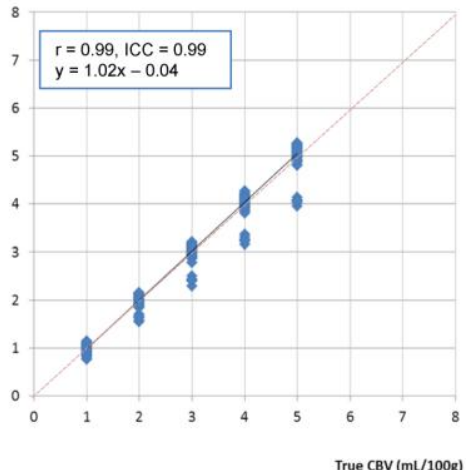

MTT

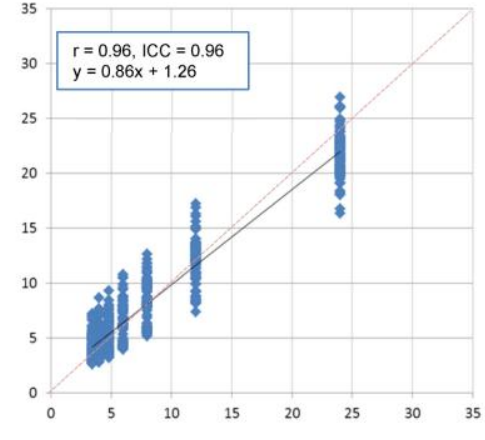

Bayesian

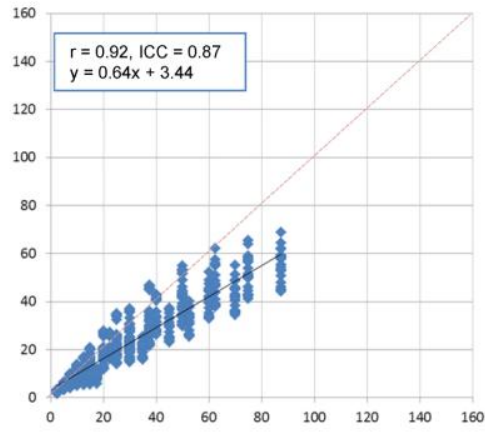

True CBF (mL/100g/min
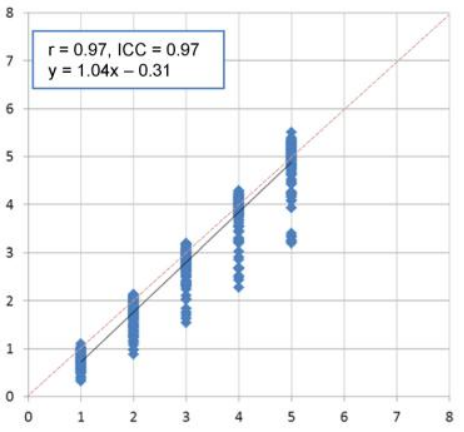

True CBV (mL/1008)
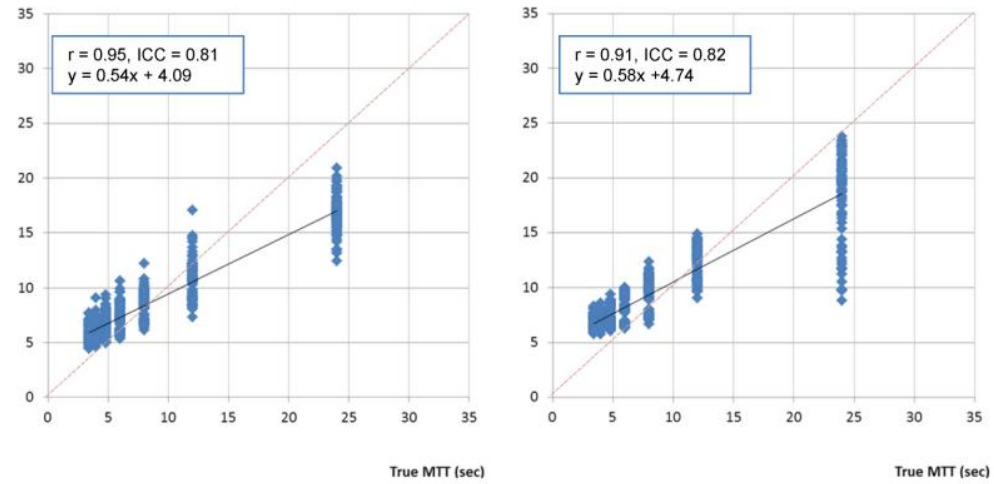

bSVD 
Table 1. Correlation and agreement of ground truth values with CBF, CBV, and MTT values generated by 3 algorithms

\begin{tabular}{|c|c|c|c|c|c|c|c|c|c|c|c|c|c|c|c|}
\hline & \multirow{2}{*}{\multicolumn{3}{|c|}{ Correlation coefficient $(r)$}} & \multirow{2}{*}{\multicolumn{3}{|c|}{$\begin{array}{l}\text { Intraclass correlation } \\
\text { coefficient (ICC) }\end{array}$}} & \multicolumn{6}{|c|}{ Regression line } & \multirow{2}{*}{\multicolumn{3}{|c|}{$\begin{array}{l}\text { Delay dependency } \\
\qquad(p \text {-value* })\end{array}$}} \\
\hline & & & & & & & \multicolumn{3}{|c|}{ Slope (a) } & \multicolumn{3}{|c|}{ Intercept (b) } & & & \\
\hline & CBF & $\mathrm{CBV}$ & MTT & CBF & $\mathrm{CBV}$ & MTT & $\mathrm{CBF}$ & CBV & MTT & $\mathrm{CBF}$ & $\mathrm{CBV}$ & MTT & CBF & CBV & MTT \\
\hline Bayesian & 0.91 & 0.99 & 0.96 & 0.90 & 0.99 & 0.96 & 1.12 & 1.02 & 0.86 & -1.00 & -0.04 & 1.26 & 0.54 & 0.54 & 0.38 \\
\hline rSVD & 0.92 & 0.97 & 0.95 & 0.87 & 0.97 & 0.81 & 0.64 & 1.04 & 0.54 & 3.44 & -0.31 & 4.09 & $<0.001$ & 0.42 & $<0.001$ \\
\hline bSVD & 0.92 & 0.98 & 0.91 & 0.91 & 0.98 & 0.82 & 1.07 & 0.92 & 0.58 & 7.37 & -0.23 & 4.74 & 0.70 & 0.42 & 0.10 \\
\hline
\end{tabular}

bSVD, block-circulant singular value decomposition; CBF, cerebral blood flow; CBV, cerebral blood volume; MTT, mean transit time; rSVD,

reformulated singular value decomposition; *, repeated-measures analysis of variance. Boldface indicates correlation of $r \geq 0.9$ or agreement of

$\mathrm{ICC} \geq 0.9$ 